\title{
El aumento de la presión del pulso es un predictor independiente de insuficiencia cardíaca en ancianos
}

Increased Pulse Pressure and Risk of Heart Failure in the Elderly.

Chae C, Pfeffer M, Glynn R, et al. JAMA, 1999;281:634-639.

\section{Objetivo}

Estudiar prospectivamente la asociación entre la presión del pulso $(P P)$, definida como la diferencia entre la tensión arterial sistólica y diastólica (TAS y TAD) y el riesgo de insuficiencia cardíaca (IC).

\section{Diseño}

Estudio prospectivo de cohortes.

Lugar

Comunidad de Boston Este, EEUU.

\section{Pacientes}

Participantes del East Boston Senior Health Project. Un censo comunitario de 1982-3 identificó 4497 residentes mayores de 65 años. El $85 \%$ fue entrevistado en su domicilio al comienzo del estudio, en 1985-6, en 1988-9 y telefónicamente desde entonces. Se obtuvo información de todos los diagnósticos de ingreso y alta hospitalaria y del status vital por bases de datos locales y nacionales (seguimiento de mortalidad del $100 \%$ de la cohorte). Excluyendo los pacientes con diagnóstico o tratamientos de IC en los últimos tres años y aquellos sin mediciones de la TA, quedaron 1621 sujetos que fueron seguidos por 3,8 años.

\section{Evaluación de factores pronósticos}

Promedio de tres tomas de la TA hechas por entrevistadores entrenados ( $P P=T A S-T A D)$. Se valoraron características sociodemográficas, historia clínica, medicación, status funcional y hábitos de salud.

\section{Medición de resultados principales}

Incidencia de IC valorada por diagnóstico de alta según la codificación ICD-9 $(n=208)$ y certificados de defunción $(n=13)$.

\section{Resultados principales}

La edad media fue de $77,9+/-5$ años ( $66 \%$ de mujeres). En el análisis multivariado* ajustando por edad, sexo, TA media, historia de enfermedad coronaria, diabetes, fibrilación auricular, valvulopatías y medicación antihipertensiva, la PP fue un predictor independiente de IC. Cada $10 \mathrm{mmHg}$ de elevación en la PP hubo un 14\% de incremento del riesgo de IC (IC95\% 5 a 24\%, p=0,003). Esta asociación parece ser más fuerte en hombres y en aquellos sin factores de riesgo de enfermedad.coronaria y no significativa en el subgrupo de pacientes que tomaba antihipertensivos (aunque el poder* para determinar esta asociación fue bajo). Aquellos en el tercilo más alto de PP (mayor a 67 $\mathrm{mmHg})$ tuvieron un $55 \%$ de riesgo de IC $(p=0,02)$ respecto al más bajo (menor a $54 \mathrm{mmHg}$ ). La PP fue más predictora que la TAS aislada y también independiente de la TAD, que por debajo de $70 \mathrm{mmHg}$ se asocia independientemente con riesgo de IC. Luego del ajuste multivariado, la PP no alcanzó a asociarse significativamente $(p=0,07)$ con los infartos miocárdicos incidentes.

\section{Conclusiones}

La PP, sencilla estimación de la rigidez arterial y la carga hemodinámica pulsátil, fue un predictor independiente de IC en esta cohorte de ancianos.

\section{COMENTARIO}

La incidencia de IC se duplica con cada década pasados los 45 años y es el principal diagnóstico hospitalario en los mayores de 65 años ${ }^{1}$. Factores de riesgo como la hipertensión han sido descriptos, pero poco se sabe de la PP, que aumenta marcadamente luego de la 5ta década. Esto se debe a la mayor rigidez arterial, que incrementa la postcarga (fuerte correlación con hipertrofia ventricular izquierda). Si bien en cohortes prospectivas de poblaciones normo e hipertensas, la PP ha mostrado ser un factor de riesgo de infarto de miocardio $^{2}$ y de mortalidad cardiovascular ${ }^{3}$, este es el único estudio que demostró que la PP es un predictor independiente y lineal de IC en una cohorte de ancianos de la comunidad. La linealidad o efecto dosis respuesta (mayor riesgo de IC a mayor PP) refuerza las conclusiones encontradas. La PP parece ser la medida individual de TA más útil para reflejar la rigidez arterial, especialmente en ancianos, pues considera el aumento de la TAS y/o la disminución de la TAD. Consistentemente, el estudio de Framingham ${ }^{4}$ encontró en la PP un mejor predictor que la TAS en hombres (especialmente mayores de 65) y no en mujeres. Además, en ancianos con hipertensión sistólica (y por ende con alta PP) el tratamiento reduce a la mitad el riesgo de IC en 4,5 años. ${ }^{5}$

Varios mecanismos pueden explicar la asociación entre PP e IC: aumento de la postcarga y de la demanda de oxígeno, deterioro de la relajación ventricular e isquemia subendocárdica, aún sin estenosis coronaria (en el presente estudio esta asociación fue independiente de enfermedad coronaria).

Aunque el uso de sistemas de codificación para la identificación de los resultados principales podría cuestionarse, estudios de validación y de admisiones al Medicare hallaron un 95,7\% de correlación del ICD-9 y los criterios diagnósticos. Aunque la internación es un razonable indicador de IC debe advertirse que no se incluyen las IC más leves.

En definitiva, la PP puede ser una herramienta simple y clínicamente útil para identificar ancianos en riesgo de IC, quienes podrían beneficiarse con tratamientos que normalicen la TA y mejoren la distensibilidad arterial.

*Ver Glosario

\section{Dr. Agustín Ciapponi}

Unidad de Medicina Familiar y Preventiva. Hospital Italiano de Buenos Aires.

\section{Referencias}

1. Congestive heart failure in the United States: a new epidemic. Bethsada, Md: National Institute Heart, Lung, and Blood Institute Information Center; September 1996. Available at http://www.nhlbi.nhi.gov/nhlbi/cardio/other/gp/CHF.htm. Accessed January 7, 1999.

2. Madhavan S, 0oi W, Cohen H, et al. Relation of pulse pressure and blood pressure reduction to the incidence of myocardial infarction. Hypertension 1994;23:395-401.

3. Benetos, A, Safar M, Rudinichi A, et al. Pulse pressure: a predictor of long term cardiovascular mortality in French male population. Hypertension 1997;30:1410-5.

4. Kannel W, Ho K, Thom T. Changing epidemiological features of cardiac failure. Br Heart J. 1994;72(suppl):S3-S9.

5. Kostis J, Davis B, Cutler J, et a! (SHEP). Prevention of Heart Failure by antihypertensive drug treatment in older persons with isolated systolic hypertension. JAMA. 1997;278:212-6. 